\title{
A novel heat transfer model and its application to information storage systems
}

\author{
Sartaj S. Ghai and Woo Tae Kim \\ Department of Chemical Engineering and Data Storage Systems Center, Carnegie Mellon University, \\ Pittsburgh, Pennsylvania 15213 and Institute for Complex Engineered Systems, Carnegie Mellon University, \\ Pittsburgh, Pennsylvania 15213 \\ Rodrigo A. Escobar and Cristina H. Amon \\ Department of Mechanical Engineering and Institute for Complex Engineered Systems, Carnegie Mellon \\ University, Pittsburgh, Pennsylvania 15213 \\ Myung S. Jhon ${ }^{\text {a) }}$ \\ Department of Chemical Engineering and Data Storage Systems Center, Carnegie Mellon University, \\ Pittsburgh, Pennsylvania 15213 and Institute for Complex Engineered Systems, Carnegie Mellon University, \\ Pittsburgh, Pennsylvania 15213
}

(Presented on 9 November 2004; published online 17 May 2005)

\begin{abstract}
Lattice Boltzmann method (LBM) based on Boltzmann transport equation is developed to simulate the nanoscale heat transport in solids. The LBM can simulate both the metals and semiconductors by properly incorporating the energy carriers. We found that boundary scattering of phonons results in an anisotropic thermal transport in nanoscale solids. The electron-phonon coupling is introduced to accurately describe the thermal behavior of nanoscale confined solids. Our numerical tool will be suitable for simulating complex multiscale systems involving multiple energy carriers with different length and time scales, and is useful in magnetic recording technology when the thermal response plays a crucial role such as for reliability of the head-disk interface and the heat assisted magnetic recording systems. () 2004 American Institute of Physics. [DOI: 10.1063/1.1853896]
\end{abstract}

\section{INTRODUCTION}

In magnetic recording systems, an emphasis has been laid on achieving higher areal density, which has forced certain characteristic lengths of the devices to nanometer scale. In heat assisted magnetic recording (HAMR) and giant magnetoresistive (GMR) head, nanoscale energy management becomes important in the operation and reliability. In HAMR system, nanometers sized hot spot is formed when a laser pulse is incident on the disk, which results in the lowering of the magnetic coercivity of the media. The energy transport from the hot spot to the lubricant film influences its adsorption/desorption and replenishment. Also, the subsequent transport of energy to the air above the disk could influence the air bearing stability. Therefore, a fundamental understanding of the subcontinuum heat conduction phenomena is critical. It is questionable whether the continuum based Fourier law accurately describe thermal behavior of this system as it does not take different energy carriers and subcontinuum effects into account whereas to examine the details of the nanoscale hot spot, we must simultaneously examine spin-electron-lattice systems. Recently, a lot of effort have been undertaken to study the sub-continuum thermal effects in solid. ${ }^{1-4}$ The Boltzmann transport equation (BTE) with the single relaxation time approximation ${ }^{5}$ is used to accurately simulate energy transport as long as the particle assumption for the heat carriers is valid. The BTE is, however, difficult to solve and, in general, a large computational

\footnotetext{
a) Author to whom correspondence should be addressed; FAX: (412) 268 -

7139; electronic mail: mj3a@andrew.cmu.edu
}

effort is required for the solution for even the simple geometries. This has led to the development of the lattice Boltzmann method (LBM), ${ }^{6}$ which, in essence, discretizes the BTE, maintaining its accuracy while reducing the computational requirement.

In this paper we are using LBM to predict transient nanoscale energy transport in the data storage area. Typically, LBM has been used as a numerical technique to solve a range of different equations with arbitrary equilibrium functions and relaxation times but in our model we are using physics based relaxation times and distribution functions. We first formulate the LBM scheme. ${ }^{7,8}$ The coupled lattice Boltzmann equations (LBEs) for electrons and phonons are simultaneously solved. Most of the previous works ${ }^{9}$ over simplified one of the two carrier's BTEs but we are using original BTEs for the two carriers. We carefully examined boundary conditions to study anisotropic heat conduction due to the nanoscale confinement. The thermal conductivity and temperature slip were calculated as functions of Knudsen number $(\mathrm{Kn})$ and specularity. A case study for the thermal profile with different energy carriers will be followed by the conclusions and discussion.

\section{THE BOLTZMANN TRANSPORT EQUATION}

The BTE with the single relaxation time approximation is given by $^{5}$

$$
\frac{\partial f}{\partial t}+\mathbf{v} \cdot \boldsymbol{\nabla} f=-\frac{f-f^{0}}{\tau}+g .
$$


Here $f, v, \tau$, and $f^{0}$ are the carrier distribution function, group velocity, relaxation time, and equilibrium distribution function, respectively. $f^{0}$ is given by the Bose-Einstein distribution $^{5,10}$ for phonons and Fermi-Dirac distribution for electrons. ${ }^{10} g$ is the phonon generation rate due to external sources such as laser heating. Equation (1) can be transformed to an equation on carrier energy density $e$ by ${ }^{11}$

$$
e(T)=\frac{1}{2 \pi} \sum_{p} \int f h \omega D_{p}(\omega) d \omega .
$$

Here $h$ is the Planck constant, $\omega$ is the frequency, and $D_{p}(\omega)$ is the density of states for carriers with polarization $p$. Then the BTE for $e$ is given by ${ }^{11}$

$$
\frac{\partial e}{\partial t}+\mathbf{v} \cdot \nabla e=-\frac{e-e^{0}}{\tau}+Q .
$$

Here $Q$ is the energy source, which is discussed in detail for the electron-phonon coupled system, in Sec. IV. The conventional definition of temperature is not valid under nonequilibrium conditions, therefore, we resort to an equivalent temperature where the total energy of carriers is equal to the total energy of the equilibrium carrier distribution at the equivalent temperature.

The phonon $p h$ and electron $e$ energy densities at their equivalent nonequilibrium temperatures are given by ${ }^{10}$

$$
\begin{aligned}
& e_{p h}\left(T_{p h}\right)=\frac{9 n_{p h} k_{B} T_{p h}^{4}}{\theta_{D}^{3}} \int_{0}^{\left(\theta_{D} / T_{p h}\right)} \frac{z^{3}}{e^{z}-1} d z, \text { and } \\
& e_{e}\left(T_{e}\right)=n_{e}\left(\frac{\pi^{2}}{2} \frac{k_{B}^{2}}{\varepsilon_{F}}\right) T_{e}^{2} .
\end{aligned}
$$

Here, $k_{B}$ is the Boltzmann constant, $\theta_{D}$ is the Debye temperature, $n_{p}$ and $n_{e}$ are the number densities of phonons and electrons, respectively, and $\varepsilon_{F}$ is the Fermi energy.

\section{THE LATTICE BOLTZMANN METHOD}

Unlike the BTE, which is a continuous equation in phase space, the LBM discretizes the space domain by defining a lattice network [Fig. 1(a)] where the energy carriers reside. Carriers propagate ballistically to neighboring lattice site and collide with carriers at that site. Time domain is discretized by restricting the carriers to travel from a lattice site to the neighboring lattice site in a given time step. Velocity space is discretized in regular intervals and only a discrete set of $\mathbf{c}_{i}$ in the main lattice directions is allowed. Then, the Eq. (3) is discretized using the forward Euler difference for the time derivative and first-order upwind scheme for the spatial derivative. The time step-lattice spacing relationship is incorporated by $\Delta \mathbf{x}_{i}=\mathbf{c}_{i} \Delta t$. This leads to the LBE with heat generation

$$
e_{i}\left(\mathbf{x}+\Delta \mathbf{x}_{i}, t+\Delta t\right)=(1-W) e_{i}(\mathbf{x}, t)+W e_{i}^{0}(\mathbf{x}, t)+Q \Delta t
$$

where $W \equiv \Delta t / \tau$ and $e_{i}(\mathbf{x}, t)$ is the discrete carrier energy distribution. Notice that the total carrier energy density is $e(\mathbf{x}, t)=\sum_{i=1}^{D} e_{i}(\mathbf{x}, t)$ ( $D$ is the number of propagation directions in the lattice). The equilibrium carrier energy distribution can be derived by assuming the isotropic distribution, (a)

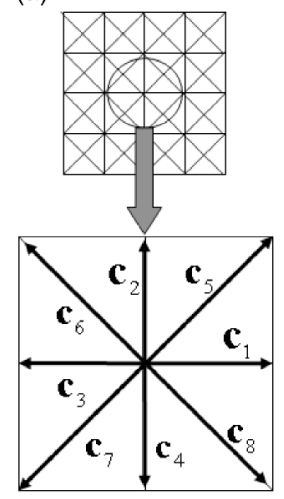

(b)

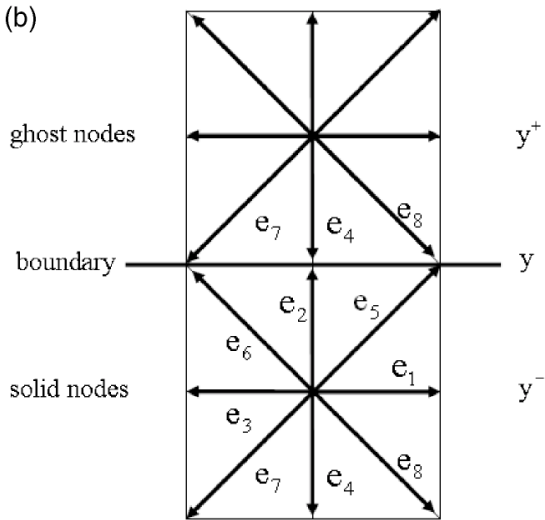

FIG. 1. (a) Two-dimensional lattice and discrete velocity vectors. (b) Schematic for the implementation of the specular and diffusive boundary conditions on the top boundary. $e_{4}\left(y^{+}\right)=P e_{2}\left(y^{-}\right)+(1-P) e_{7}^{0}\left(y^{+}\right), e_{7}\left(y^{+}\right)=P e_{6}\left(y^{-}\right)$ $+(1-P) e_{4}^{0}\left(y^{+}\right)$, and $e_{8}\left(y^{+}\right)=P e_{5}\left(y^{-}\right)+(1-P) e_{8}^{0}\left(y^{+}\right) . e_{4}^{0}, e_{7}^{0}$, and $e_{8}^{0}$ are evaluated using the temperature obtained from the energy conservation. Specularity $P$ is the fraction of carriers which undergo specular reflection at the boundary.

$e_{i}^{0}(\mathbf{x}, t)=e(\mathbf{x}, t) / D$. This definition inherently guarantees energy conservation. Temperature distribution can be found from Eq. (4) once Eq. (5) is solved. Phonon LBE is solved for the case of a hot-spot generation and we found that, as the hot-spot diameter becomes smaller than the phonon mean free path, the LBE captures the subcontinuum effect of higher temperature rise but the Fourier equation fails to do so.

\section{ELECTRON-PHONON COUPLING}

We examined heating of thin metal films via laser pulse that energize the electrons. Electrons subsequently transfer the energy to phonons via coupling between them. To study this phenomenon, two coupled LBEs (Eq. (5), one each) for electrons and phonons, are simultaneously solved for different length and time domains using multigrid simulation technique. For electrons $Q=Q^{\prime}-G\left(T_{e}-T_{p h}\right)$ and for phonons $Q$ $=G\left(T_{e}-T_{p h}\right)$, where $Q^{\prime}$ is the power density deposited by the external source, and $G$ is the coupling constant. The coupling term between the two LBEs will act as a channel of thermal transport between the two carriers and will help in the energy relocation.

To examine the effect of the $G$, we performed a laser heating simulation for a typical metal. A laser pulse incidence at the center of a solid is simulated while considering both electrons and phonons as energy carriers. The represen-

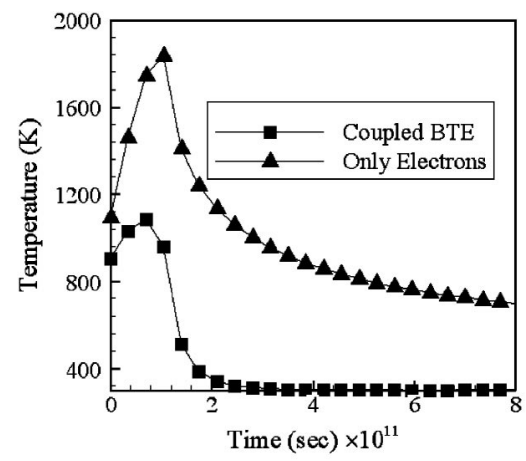

FIG. 2. Transient electron temperature profile at the center of a hot spot. 


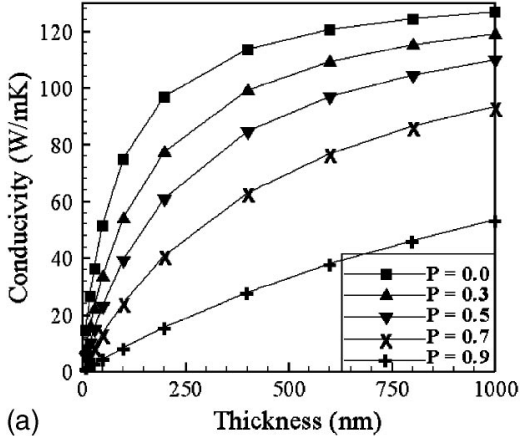

(a)

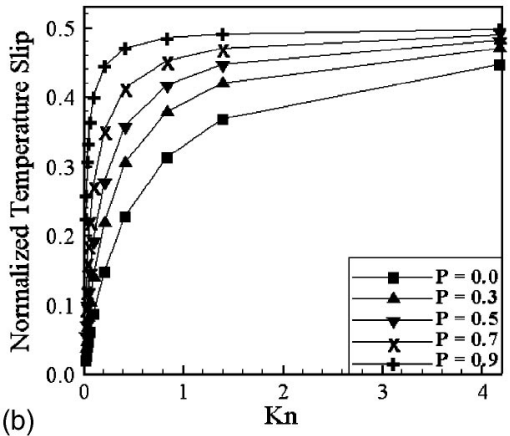

FIG. 3. LBM simulation results at five different $P$ values for silicon thin film: (a) thermal conductivity vs thickness and (b) temperature slip (normalized by total applied temperature difference across the thin film) vs $\mathrm{Kn}$. tative electron and phonon mean free paths and relaxation times were taken as 10 and $450 \mathrm{~nm}$ and 0.1 and $70 \mathrm{ps}$, respectively. $Q^{\prime}$ is taken as $10^{18} \mathrm{~W} / \mathrm{m}^{3}$. For $G=2$ $\times 10^{13} \mathrm{~W} / \mathrm{m}^{3} \mathrm{~K}$, even though phonons show a low temperature rise, owing to their (about two orders of magnitude) higher specific heat, both carriers act as primary modes of energy transport. As $G$ decreases from $2 \times 10^{13}$ to $2 \times 10^{12}$, the electron temperature shows similar profile with a little increase in temperature and remains almost identical from $G=2 \times 10^{12}$ to $2 \times 10^{11}$. However, phonon temperature profile shows a significant change as $G$ varies. As $G$ is decreased to $2 \times 10^{12}$, the energy is primarily transferred from the electrons to the phonons when the pulse is on and the electrons are at a very high temperature. As $G$ is further decreased, there is almost no energy transfer to the phonons and electrons alone acts as the primary mode of energy transfer.

To examine the effect of both energy carriers, we simulated a hot spot in solid while considering only electrons, only phonons, and both electrons and phonons as the energy carriers. The values of parameters used in this case are same as the values used previously. The transient profile of electron temperature at the center of the hot spot is presented in Fig. 2. It is observed that the coupled simulation exhibits a smaller electron temperature rise, than the single carrier simulation, due to the increased specific heat of the system because of the presence of two channels of energy transport. It is also observed that the electron temperature relaxes much faster due to the presence of an additional channel, phonons, of heat transfer having a high specific heat which results in a higher effective conductivity. A good understanding of the hot-spot phenomena is crucial for HAMR system. LBM can provide an accurate description of the hot spot and thus helps in the operation and design of the HAMR system.

\section{BOUNDARY CONDITIONS}

Unlike bulk solids, it has been observed that the boundary conditions strongly affect the thermal transport in nanoscale confined solids. When the characteristic length of the solid becomes smaller or on the order of the bulk mean free path of the carriers, the boundary effects become significant and they govern the effective mean free path of the carriers and thus affect the transport properties of the solid. The confinement leads to anisotropic conductivity in otherwise isotropic solids.

At the boundary, energy carriers can be scattered either specularly or diffusively. For the specular reflection, the re- flected angle of an energy carrier is set equal to the incident angle. For the diffusive reflection, an energy carrier is reflected at some random angle that is uncorrelated with its angle of incidence while conserving the energy transferred to the boundary. A real surface is treated as a combination of both mechanisms. We have incorporated these boundary conditions using the ghost nodes [Fig. 1(b)]. and observed that the thermal conductivity and temperature slip at the boundaries depend strongly on the $P$ and the dimensions of the film via $\mathrm{Kn}$. Some representative results for thermal conductivity and temperature slip for silicon are shown in Fig. 3.

\section{CONCLUSION AND DISCUSSION}

A discrete mathematical model, LBM, is presented to simulate nanoscale heat transfer in solids. The effect of the electron-phonon coupling on the thermal modeling of solids is discussed and its importance to the data storage systems, e.g., HAMR and GMR heads is elucidated. The coupling term is varied in the simulations to present its importance for the thermal transport in the head and media of hard-disk drive. A significantly different thermal behavior is obtained while considering either one of the energy carriers. The correct treatment of the boundary conditions and anisotropic conductivity and temperature slip for silicon thin film are examined. The model incorporating a realistic threedimensional hot-spot simulation is required to examine the actual thermal phenomena, which is crucial in HAMR technology as it governs the rate of cooling of the hot spot by transferring the energy to the substrate, lubricant film, and the air bearing and effects the adsorption/desorption of the lubricant as well as the stability of the air bearing.

${ }^{1}$ M. S. Jhon, Adv. Chem. Phys. 129, 1 (2004).

${ }^{2}$ A. A. Joshi and A. Majumdar, J. Appl. Phys. 74, 31 (1993).

${ }^{3}$ G. Chen, J. Heat Transfer 124, 320 (2002).

${ }^{4}$ S. Narumanchi, J. Y. Murthy, and C. H. Amon, J. Heat Transfer 125, 896 (2003).

${ }^{5}$ C. Cercignani, The Boltzmann Equation and Its Application (Springer, New York, NY, 1988).

${ }^{6}$ S. Succi, The Lattice-Boltzmann Equation for Fluid Dynamics and Beyond (Clarendon, Oxford, 2001)

${ }^{7}$ S. S. Ghai, R. A. Escobar, C. H. Amon, and M. S. Jhon, InterPack 200335258 (2003).

${ }^{8}$ R. A. Escobar, S. S. Ghai, M. S. Jhon, and C. H. Amon, IMECE200341522 (2003).

9. Lai and A. Majumdar, J. Appl. Phys. 79, 7353 (1996).

${ }^{10}$ N. W. Ashcroft and N. D. Mermin, Solid State Physics (Harcourt, Fort Worth, TX, 1976).

${ }^{11}$ W. Zhang and T. S. Fisher, IMECE2002-32122 (2002). 